\title{
COHOMOLOGY OF EQUIVARIANT MAPS
}

\author{
BY \\ J. DUGUNDJI
}

1. Introduction. This paper is concerned with the equivariant cohomology groups, and with the homomomorphisms of these groups induced by equivariant maps.

Let the group $W$ operate on a complex $K$, and denote by $h_{n}(K)$ the group of integral $n$-chains modulo the $n$-boundaries. $W$ operates on $h_{n}(K)$ in the obvious manner, and an equivariant $n$-cohomology class $e_{W}^{n}(K)$ $\in H_{W}^{n}\left(K ; h_{n}(K)\right)$ is singled out in 3.1 ; this class is shown to be the zero class if and only if $H_{W}^{n}(K ; G)=0$ for all groups $G$ with operators $W$. Further consequences of its vanishing are given in $\$ 3$. Defining the $n$-degree of an equivariant map $f: P \rightarrow K$ to be $f^{+} e_{W}^{n}(K)$, it is proved (4.3) that the $n$-degree completely determines the homomorphisms $H_{W}^{n}(K ; G) \rightarrow H_{W}^{n}(P ; G)$ induced by $f$ for all groups $G$ with operators $W$.

This theory for complexes does not extend invariantly, even to polytopes $P$, since $h_{n}(P)$ is not an invariant of the simplicial subdivision used. This difficulty is overcome, and the theory extended to arbitrary spaces; by working in the singular complex of the space. Although $H_{W}^{n}\left(Y ; h_{n}(Y)\right)$ thus becomes a topological invariant of $Y$ and $W$, the drawback is that its explicit calculation, and that of $e_{W}^{n}(Y)$, is not practicable. However, in the case that $Y$ is a polytope, there is a fixed subgroup $\Delta_{W}^{n} \subset H_{W}^{n}\left(Y ; h_{n}(Y)\right)$, reducing to $H^{n}\left(Y ; H_{n}(Y)\right)$ whenever $W$ operates simply, which can be computed directly from any simplicial subdivision. The group $\Delta_{W}^{n}$, and conditions under which $e_{W}^{n}(Y)$ lies in $\Delta_{W}^{n}$, are given in $\S 5$ and the topological considerations in $\S 6$.

In $\S I$, three applications are given. The first, $\S 7$, is a universal coefficient theorem for the homomorphisms of the equivariant cohomology groups induced by equivariant maps, which is analogous to that in $[1$, p. 238]. The second application, $\S 8$, is to obtain a universal coefficient theorem for $H_{W}^{n}(K ; G)$, valid whenever $C_{n-1}(K)$ is $W$-free; the groups $h_{n}(K)$ and $h_{n-1}(K)$ play an important role here. The third application, in $\S 9$, is to the obstruction cocycle: the mechanism whereby the equivariant map $\varphi: Q \rightarrow Y$ determines the set of all (homology and homotopy) $(n+1)$-obstructions that can possibly be met by any of its extensions over $Q \cup P^{n}$ is given.

I.

2. Preliminaries and notation. An abelian group $G$ together with a multiplicative (not necessarily abelian) group $W$ of (left) operators $\left({ }^{1}\right)$ is called a

Received by the editors October 29, 1956 and, in revised form, December 28, 1956.

(1) That is, for each $w \in W$ an autmorphism $w: G \rightarrow G$ is given such that $1 g=g$ and $w_{1} w_{2}(g)$ $=w_{1}\left(w_{2} g\right)$ for all $g \in G, w_{1}, w_{2} \in W$. $W$ operates simply on $G$ if $w g=g$ for all $w \in W, g \in G$. 
$W$-group; a subgroup $H \subset G$ is $W$-stable if $w H \subset H$ for each $w \in W$. A homomorphism $h: G \rightarrow G^{\prime}$ into another $W$-group $G^{\prime}$ is called a $W$-homomorphism if $w h=h w$ for all $w \in W$. The following result will be used frequently:

2.1. In the diagram $M \leftarrow^{i} G \rightarrow^{i} H, M, G, H$ are $W$-groups and $i, j W$ homomorphisms. If (a) $: j$ is onto and (b): Kernel $j \subset$ Kernel $i$, then there is a $W$-homomorphism $k: H \rightarrow M$ with $k j=i$.

In fact, by (a), each $h \in H$ has form $j(g)$ for some $g \in G$. Define $k(h)=i(g)$; $k(h)$ is uniquely determined because of (b) and is the required homomorphism.

A $W$-group $G$ is $W$-free if there is a set $\left\{g_{\mu}\right\}$ of elements of $G$ such that $\left\{w g_{\mu}\right\}$, for all $\mu$ and all $w \in W$, is a set of free generators for $G$; the collection $\left\{g_{\mu}\right\}$ itself is called a $W$-basis for $G$. It is well-known that $[3$, p. 55].

2.2. In the diagram $G \rightarrow^{i} H \leftarrow^{i} F, G, H, F$ are $W$-groups and $i, j W$-homomorphisms. If (a) $j$ is onto and (b): $F$ is $W$-free, then there is a $W$-homomorphism $k: F \rightarrow G$ with $j k=i$.

A $W$-group $G$ is the $W$-direct sum of the subgroups $G^{\prime}, G^{\prime \prime}$, if $G$ is the direct sum $G^{\prime} \oplus G^{\prime \prime}$ of $G^{\prime}$ and $G^{\prime \prime}$, and both $G^{\prime}, G^{\prime \prime}$, are $W$-stable.

Let $K$ be an augmentable closure-finite cell complex; its $n$-skeleton will be denoted by $K^{n}$. The free abelian group $C_{n}(K)$ of finite integral $n$-chains of $K$ contains the usual subgroups $Z_{n}(K)$ and $B_{n}(K)$ of cycles and boundaries respectively, and leads to the $n$th homology group $H_{n}(K)=Z_{n}(K) / B_{n}(K)$. It is well-known that $[4$, p. 154].

2.3. (a): $C_{n}(K)$ is the direct sum $Z_{n}(K) \oplus M$, where $M \approx B_{n-1}(K)$.

(b): $H_{n}(K)$ is free if and only if $B_{n}(K)$ is a direct summand of $Z_{n}(K)$.

If $K$ has a group of operators $\left({ }^{2}\right) W$, it is important to observe that, even though $Z_{n}(K)$ is $W$-stable, the subgroup $M$ in 2.3(a) need not be, so that the direct sum decomposition of $C_{n}(K)$ is in general not a $W$-direct sum.

Let $K$ have operators $W$, let $L$ be a segregated $\left({ }^{3}\right)$ subcomplex, and let $G$ be a $W$-group. The group $C_{W}^{n}(K, L ; G)$ of equivariant $(n, G)$-cochains $\bmod L$ is the group of all $W$-homomorphisms $f: C_{n}(K) \rightarrow G$ satisfying $f\left(C_{n}(L)\right)=0$. The coboundary $\delta f$ of the cochain $f$ is the $W$-homomorphism $\delta f=f \partial: C_{n+1}(K)$ $\rightarrow G$; this leads in the usual fashion to the groups of equivariant cocycles $Z_{W}^{n}(K, L ; G)$ and coboundaries $B_{W}^{n}(K, L ; G)$, and to the equivariant $n$th cohomology group $H_{W}^{n}(K, L ; G)$. Omission of the subscript $W$ will mean the $W$ operates simply on $K$. The cohomology class of an equivariant cocycle $f$ is written $[f]$.

The following is evident:

2.4. Let $G, J$, be $W$-groups, and $K$ a complex with operators $W$. A $W$ -

(2) A multiplicative (not necessarily abelian) group $W$ operates on a complex $K$ if for each $w \in W$ there is given an automorphism $w: C_{n}(K) \rightarrow C_{n}(K)$, for each dimension $n$, such that $w \partial=\partial w, 1 c=c$ and $w_{1} w_{2}(c)=w_{1}\left(w_{2} c\right)$ for every $c \in C_{n}, w, w_{1}, w_{2} \in W$ and every $n \geq 0$. If each $w$ is a simplicial map of $K$ in itself, $W$ is said to operate simplicially on $K$; if $w c=c$ for all $c, W$ operates simply.

(8) $L$ is segregated in $K$ if for each $w \in W$ and each integral chain $c$ in $L$ or in $K-L$, wc is also in $L$ or in $K-L$. 
homomorphism $p: G \rightarrow J$ induces a homomorphism

$$
p^{*}: H_{W}^{n}(K ; G) \rightarrow H_{W}^{n}(K ; J)
$$

by setting $p^{\sharp}[f]=[p f]$ for each $f \in Z_{W}^{n}(K ; G)$.

Since the homomorphism $H_{W}^{n}(P, \cdot) \rightarrow H_{W}^{n}(K, \cdot)$ induced by an equivariant chain $\operatorname{map}\left({ }^{4}\right) \mu$ of $K$ into another complex $P$ with operators $W$ is $\mu^{+}[f]$ $=[f \mu]$, the diagram

$$
\begin{array}{crr}
H_{W}^{n}(P ; G) & \stackrel{p^{*}}{\rightarrow} H_{W}^{n}(P ; J) \\
\mu^{+} \downarrow & \quad \downarrow \mu^{+} \\
H_{W}^{n}(K ; G) & \stackrel{p^{*}}{\rightarrow} H_{W}^{n}(K ; J)
\end{array}
$$

is commutative.

3. The $(n, W)$-universal cohomology class of a complex. Let $K$ be a complex, and for each $n \geqq 0$ denote the factor group $C_{n}(K) / B_{n}(K)$ of the integral $n$-chains modulo the $n$-boundaries $\left(^{5}\right)$ by $h_{n}(K)$; according to $2.3, h_{n}(K)$ $=H_{n}(K) \oplus M$, where $M \approx B_{n-1}(K)$.

Now let $K$ have operators $W$. Each chain transformation $w \in W$ induces an automorphism $w$ of $h_{n}(K)$ and of $H_{n}(K)$; with this action of $W, h_{n}(K)$ and $H_{n}(K)$ will be regarded as $W$-groups. $H_{n}(K)$ is a $W$-stable subgroup of $h_{n}(K)$, but is not necessarily a $W$-direct summand, unless $W$ operates simply on $K$.

Take the $W$-group $h_{n}(K)$ as coefficient group for the $n$-cochains. The natural projection

$$
\eta: C_{n}(K) \rightarrow C_{n}(K) / B_{n}(K)
$$

is clearly a $W$-homomorphism, and $\eta\left(B_{n}\right)=0$; thus $\eta \in Z_{W}^{n}\left(K ; h_{n}(K)\right)$.

3.1. Definition. $\eta$ is called the $(n, W)$-universal cocycle of $K$; its cohomology class $e_{W}^{n}(K) \in H_{W}^{n}\left(K ; h_{n}(K)\right)$ is the $(n, W)$-universal cohomology $\operatorname{class}\left({ }^{6}\right)$ of $K$.

The following theorem makes precise the sense of "universal":

3.2. THEOREM. For any $W$-group $G$ and any $\alpha \in H_{W}^{n}(K ; G)$ there is a $W$ homomorphism $p: h_{n}(K) \rightarrow G$ such that $p^{\sharp} e_{W}^{n}(K)=\alpha$, where $p^{\sharp}$ is the homomorphism $H_{W}^{n}\left(K ; h_{n}(K)\right) \rightarrow H_{W}^{n}(K ; G)$ induced by $p$.

(4) If $K, P$ are complexes with operators $W$, an equivariant chain map $\mu: K \rightarrow P$ is a set of $W$-homomorphisms $\mu: C_{n}(K) \rightarrow C_{n}(P)$, one for each dimension, such that $\mu \partial=\partial \mu$.

(5) If $K$ is a polytope, $h_{n}(K)$ is clearly not a topological invariant nor an invariant of simplicial subdivision. This matter, together with the extension of the theory to arbitrary spaces, will be considered in $\$ 6$.

(6) The construction of this cohomology class, for use in an entirely different connection, was first brought to my attention by W. Hurewicz. 
This theorem is an immediate consequence of 2.4 and the following

3.3. Lemma. Let $G$ be a $W$-group, and $g \in Z_{W}^{n}(K ; G)$. Then there is a $W$ homomorphism $p: h_{n}(K) \rightarrow G$ with $p \eta=g$.

Proof. In the diagram

$$
G \stackrel{g}{\longleftarrow} C_{n}(K) \stackrel{\eta}{\rightarrow} h_{n}(K)
$$

$\eta$ is onto and kernel $\eta=B_{n}$ C kernel $g$ because $g$ is a cocycle; 2.1 thus applies.

The vanishing of $e_{W}^{n}(K)$ and some of its consequences will now be considered. It is immediate from 3.2 that

3.4. Theorem. $e_{W}^{n}(K)=0$ if and only if $H_{W}^{n}(K ; G)=0$ for all $W$-groups $G$. In particular, $e_{W}^{n}(K)=0$ if and only if $H_{W}^{n}\left(K ; h_{n}(K)\right)=0$.

In the case that $W$ operates simply on $K$, two further conclusions will be given, and then applied to the general case.

3.5. Theorem. Let $W$ operate simply on $K . H^{n}\left(K ; h_{n}(K)\right)=0$ if and only if both $H_{n}(K)=0$ and $H_{n-1}(K)$ are free.

Proof. By the universal coefficient theorem [4, p. 161], $H^{n}\left(K ; h_{n}(K)\right)=0$ if and only if both $\operatorname{Hom}\left(H_{n}, h_{n}\right)=0$ and the injection $i^{+}$: Hom $\left(Z_{n-1}, h_{n}\right)$ $\rightarrow \operatorname{Hom}\left(B_{n-1}, h_{n}\right)$ is onto. Since $H_{n} \subset h_{n} \operatorname{Hom}\left(H_{n}, h_{n}\right)=0$ if and only if $H_{n}(K)=0$. If $H_{n-1}$ is free, then (2.3) $B_{n-1}$ is a direct summand of $Z_{n-1}$ and $i^{+}$is onto. For the converse, identify $B_{n-1}$ as a direct summand of $h_{n}$ and let $j: B_{n-1} \rightarrow h_{n}$ be the injection; since $i^{+}$is onto, $j$ extends to $j: Z_{n-1} \rightarrow h_{n}$. Letting $r: h_{n} \rightarrow B_{n-1}$ be the retraction, $r j$ is a retraction of $Z_{n-1}$ onto $B_{n-1}$; as is well known, this implies that $B_{n-1}$ is a direct summand of $Z_{n-1}$ hence 2.3 shows $H_{n-1}$ is free.

3.6. Theorem. Let $W$ operate simply on $K . H^{n}\left(K ; h_{n}(K)\right)=0$ if and only if both $H^{n}(K ; G)=0$ and $H_{n}(K ; G)=0$ for all coefficient groups $G$.

Proof. Because of 3.4, only the implication that $H_{n}(K ; G)=0$ for all $G$ need be proved. Using 3.5, $H_{n}(K) \otimes G=$ Tor $\left(H_{n-1}, G\right)=0$, so that the result follows from the universal coefficient theorem.

Returning to the case where $W$ does not necessarily operate simply on $K$, observe that each equivariant cocycle is also an ordinary cocycle, and that this correspondence [2, p. 383] induces a homomorphism $u: H_{W}^{n}(K ; G)$ $\rightarrow H^{n}(K ; G)$. It is immediate from the definitions that $u e_{W}^{n}(K)=e^{n}(K)$, the universal $n$-cohomology class when $W$ operates simply. The following complement to 3.4 is then immediate:

3.7. Theorem. If $e_{W}^{n}(K)=0$, then both $H_{n-1}(K)$ is free and $H^{n}(K ; G)$ $=H_{n}(K ; G)=0$ for all groups $G$. 


\section{The $(n, W)$-degree of an equivariant chain map.}

4.1. Definition. Let $K$ and $P$ be complexes with operators $W$, and $\varphi: P \rightarrow K$ an equivariant chain map. The $(n, W)$-degree $e_{W}^{n}(\varphi)$ of $\varphi$ is the element $\varphi^{+} e_{W}^{n}(K) \in H_{W}^{n}\left(P ; h_{n}(K)\right)$.

4.2. REMARK. The degree $e_{W}^{n}(\varphi)$ can also be obtained as follows: $\varphi$ induces a $W$-homomorphism $\varphi_{+}: h_{n}(P) \rightarrow h_{n}(K)$ which in turn gives a homororphism $\left(\varphi_{+}\right)^{\sharp}: H_{W}^{n}\left(P ; h_{n}(P)\right) \rightarrow H_{W}^{n}\left(P ; h_{n}(K)\right)$; the commutativity of

$$
\begin{array}{cr}
C_{n}(P) \stackrel{\eta^{\prime}}{\rightarrow} & h_{n}(P) \\
\varphi \downarrow & \downarrow \\
C_{n}(K) \underset{\eta}{\rightarrow} & h_{n}(K)
\end{array}
$$

implies at once that $\varphi^{+} e_{W}^{n}(K)=\left(\varphi_{+}\right)^{*} e_{W}^{n}(P)$. As a consequence, maps inducing the same $W$-homomorphism $h_{n}(P) \rightarrow h_{n}(K)$ have the same $(n, W)$-degree; the converse is not necessarily true (see 5.4).

4.3. TheOREM. The $(n, W)$-degree of $\varphi$ determines the homomorphisms $\varphi^{+}: H_{W}^{n}(K ; G) \rightarrow H_{W}^{n}(P ; G)$ for all $W$-groups $G$. In particular, for two equivariate chain maps $\varphi, \psi: P \rightarrow K, e_{W}^{n}(\varphi)=e_{W}^{n}(\psi)$ if and only if $\varphi^{+}, \psi^{+}: H_{W}^{n}(K ; G)$ $\rightarrow H_{W}^{n}(P ; G)$ are the same for all $W$-groups $G$.

Proof. Let $\alpha \in H_{W}^{n}(K ; G)$; using 3.2 , there is a $W$-homomorphism $p: h_{n}(K) \rightarrow G$ with $p^{\sharp} e_{W}^{n}(K)=\alpha$. It will be shown that the same coefficient group homomorphism $p^{\sharp}$ applied to $H_{W}^{n}\left(P ; h_{n}(K)\right)$ sends $e_{W}^{n}(\varphi)$ to $\varphi^{+} \alpha$. In fact, if $\alpha=[f]$ find $p$ with $p \eta=f$; then $\varphi^{+} \alpha=[f \varphi]=[p \eta \varphi]=p^{\sharp}[\eta \varphi]=p^{\sharp} e_{W}^{n}(\varphi)$. The theorem now follows at once.

Further consequences of the equality of the degrees of two maps will now be given; as in $\S 3$, the case that $W$ operates simply is considered first.

4.4. Theorem. Let $W$ operate simply on $K$ and $P$, and let $\varphi, \psi: P \rightarrow K$ be two chain maps. Then $e^{n}(\varphi)=e^{n}(\psi)$ if and only if $\varphi, \psi$ induce identical homomorphisms $H_{n}(P) \rightarrow H_{n}(K)$ and $H^{n}(K ; G) \rightarrow H^{n}(P ; G)$ for all groups $G$.

Proof. From 4.3 with $W$ operating simply, only that $e^{n}(\varphi)=e^{n}(\psi)$ implies the homomorphisms $H_{n}(P) \rightarrow H_{n}(K)$ are identical need be proved. Observe $e^{n}(\varphi)=e^{n}(\psi)$ means $\eta \varphi-\eta \psi=\mu \partial$ for some homomorphism $\mu: C_{n-1}(P) \rightarrow h_{n}(K)$. Consequently for any $n$-cycle $z \in Z_{n}(P), \eta(\varphi(z)-\psi(z))=\mu \partial z=0$, that is, $\varphi(z)$ is homologous to $\psi(z)$ in $K$; the homomorphisms $\varphi_{+}, \psi_{+}: H_{n}(P) \rightarrow H_{n}(K)$ are therefore identical.

4.5. Theorem. Let $W$ operate simply on $K$ and $P$, and let $\varphi: P \rightarrow K$ be a chain map. If $H_{n-1}(P)$ is free, the $n$-degree of $\varphi$ determines the homomorphisms $\varphi_{+}: H_{n}(P ; G) \rightarrow H_{n}(K ; G)$ for every $G$.

Proof. According to the universal coefficient theorem, the diagram 


$$
\begin{aligned}
& 0 \rightarrow H_{n}(P) \otimes G \stackrel{i}{\rightarrow} H_{n}(P ; G) \stackrel{j}{\rightarrow} \text { Tor }\left(H_{n-1}(P) ; G\right) \rightarrow 0 \\
& \psi_{+}^{\prime} \downarrow\left\{\varphi_{+}^{\prime}\right\} \quad \psi_{+} \downarrow\left\{\varphi_{+}\right\} \\
& 0 \rightarrow H_{n}(K) \otimes G \underset{i^{\prime}}{\rightarrow} H_{n}(K ; G) \underset{j^{\prime}}{\rightarrow} \operatorname{Tor}\left(H_{n-1}(K) ; G\right) \rightarrow 0
\end{aligned}
$$

is exact along each horizontal and commutative in each square. Since $H_{n-1}(P)$ is free, Tor $\left(H_{n-1}(P) ; G\right)=0$. If $e^{n}(\varphi)=e^{n}(\psi)$ then by $4.4 \varphi_{+}^{\prime}=\psi_{+}^{\prime}$ and this immediately results in $\varphi_{+}=\psi_{+}$.

It is easy to construct examples showing that the condition " $H_{n-1}(P)$ free" cannot be removed.

As is readily verified, the homomorphism

$$
u: H_{W}^{n}\left(P ; h_{n}(K)\right) \rightarrow H^{n}\left(P ; h_{n}(K)\right)
$$

gives $u e_{W}^{n}(\varphi)=e^{n}(\varphi)$. The following complement to 4.3 is then immediate:

4.6. Theorem. Let $P, K$ be complexes with operators $W$, and $\varphi, \psi: P \rightarrow K$ equivariant chain maps. If $e_{W}^{n}(\varphi)=e_{W}^{n}(\varphi)$ then $\varphi, \psi$ induce identical homomorphisms $H_{n}(P) \rightarrow H_{n}(K)$ and $H^{n}(K ; G) \rightarrow H^{n}(P ; G)$ for all groups $G$. If, in addition, $H_{n-1}(P)$ is free, then the induced homomorphisms of all homology groups, regardless of coefficients used, are the same.

5. Location of the $(n, W)$-degree of a chain map. Since $H_{n}(K)$ is a $W$-stable subgroup of $h_{n}(K)$, the identity map $H_{n}(K) \rightarrow h_{n}(K)$ is a $W$-homomorphism.

5.1. Definition. The image of $H_{W}^{l}\left(P, Q ; H_{n}(K)\right)$ in $H_{W}^{l}\left(P, Q ; h_{n}(K)\right)$ under the homomorphism $\gamma^{\sharp}$ induced by the injection $\gamma: H_{n}(K) \rightarrow h_{n}(K)$ is written $\Delta_{W}^{l}\left(P, Q ; H_{n}(K)\right)$.

In this section, some conditions under which the $(n, W)$-degree of a chain map $\varphi: P \rightarrow K$ lies in $\Delta_{W}^{n}\left(P ; H_{n}(K)\right)$ will be given $\left({ }^{7}\right)$.

5.2. Theorem. Let $P, K$ have operators $W$, and let $\varphi: P^{n+1} \rightarrow K^{n+1}$ be an equivariant chain map. Assume (a): $C_{n-1}(P)$ is $W$-free and (b): The $W$-homomorphism $\varphi_{+}: h_{n-1}(P) \rightarrow h_{n-1}(K)$ induced by $\varphi$ factors into $h_{n-1}(P) \rightarrow F \rightarrow h_{n-1}(K)$ where $F$ is $W$-free. Then $e_{W}^{n}(\varphi) \in \Delta_{W}^{n}\left(P ; H_{n}(K)\right)$.

Proof. Consider the diagram

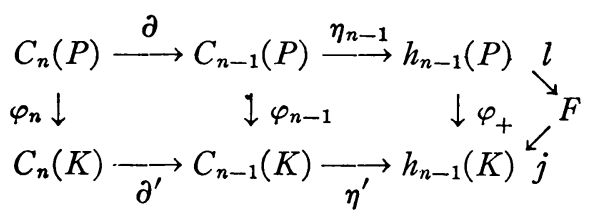

where commutativity holds in each square. Since $F$ is $W$-free and $\eta^{\prime}$ is onto, 2.2 gives a $W$-homomorphism $q: F \rightarrow C_{n-1}(K)$ with $j=\eta^{\prime} q$. The $W$-homo-

(7) The utility of the group $\Delta_{W}^{*}$ will appear later (cf. Footnote 5). 
morphism $\varphi_{n-1}-q l_{n-1}$ is such that $\eta^{\prime} \varphi_{n-1}-\eta^{\prime} q l_{n-1}=0$, hence sends $C_{n-1}(P)$ into $B_{n-1}(K)$. Since $C_{n-1}(P)$ is $W$-free and $\partial^{\prime}$ is onto $B_{n-1}(K)$ apply 2.2 again to find $p: C_{n-1}(P) \rightarrow C_{n}(K)$ with $\varphi_{n-1}-q \eta_{n-1}=\partial^{\prime} p$. Finally, consider

$$
\varphi_{n}-p \partial: C_{n}(P) \rightarrow C_{n}(K) \text {. }
$$

Since $\partial^{\prime}\left(\varphi_{n}-p \partial\right)=\partial^{\prime} \varphi_{n}-\partial^{\prime} p \partial=\partial^{\prime} \varphi_{n}-\varphi_{n-1} \partial+q l \eta_{n-1} \partial=0, \varphi_{n}-p \partial$ assumes values in $Z_{n}(K)$ only. Letting $\eta: C_{n}(K) \rightarrow h_{n}(K)$ be the natural homomorphism, it follows that $\eta \varphi_{n}-\eta p \partial$ is an $n$-cocycle in $P$ with values in $H_{n}(K)$. Since $\eta p: C_{n-1}(P) \rightarrow h_{n}(K)$, this means $e_{W}^{n}(\varphi)$ is cohomologous in $H_{W}^{n}\left(P ; h_{n}(K)\right)$ to a cocycle in $\Delta_{W}^{n}\left(P ; H_{n}(K)\right)$ and proves the assertion.

The following is a partial converse to 5.2:

5.3. Theorem. Let $P, K$ have operators $W$, and let $\varphi: P^{n+1} \rightarrow K^{n+1}$ be an equivariant chain map. Assume (a): Both $C_{n-1}(P)$ and $C_{n-1}(K)$ are $W$-free and (b): $e_{W}^{n}(\varphi)$ lies in $\Delta_{W}^{n}\left(P ; H_{n}(K)\right)$. Then the homomorphism $h_{n-1}(P) \rightarrow h_{n-1}(K)$ induced by $\varphi$ factors into $h_{n-1}(P) \rightarrow F \rightarrow h_{n-1}(K)$ where $F$ is $W$-free.

Proof. The hypothesis gives $\eta \varphi_{n}-q \partial: C_{n}(P) \rightarrow H_{n}(K)$ for some $W$-homomorphism $q: C_{n-1}(P) \rightarrow h_{n}(K)$. From the diagram

$$
C_{n}(K) \stackrel{\eta}{\rightarrow} h_{n}(K) \stackrel{q}{\leftarrow} C_{n-1}(P)
$$

there is, according to 2.2 , a homomorphism $p: C_{n-1}(P) \rightarrow C_{n}(K)$ with $q=\eta p$. Since $\eta \varphi_{n}-q \partial=\eta\left(\varphi_{n}-p \partial\right)$ it follows that $\varphi_{n}-p \partial$ assumes values only in $Z_{n}(K)$ and consequently $\varphi_{n-1}-\partial^{\prime} p$ annihilates $B_{n-1}(P)$. Consider now the diagram

$$
\begin{array}{rr}
C_{n-1}(P) \stackrel{\eta_{n-1}}{\longrightarrow} h_{n-1}(P) \\
\varphi_{n-1}-\partial^{\prime} p \downarrow & \downarrow \varphi_{+} \\
C_{n-1}(K) \underset{\eta^{\prime}}{\longrightarrow} & h_{n-1}(K)
\end{array}
$$

This diagram commutes, since $\eta^{\prime}\left(\varphi_{n-1}-\partial^{\prime} p\right)=\eta^{\prime} \varphi_{n-1}=\varphi_{+} \eta_{n-1}$. Because $\varphi_{n-1}-\partial^{\prime} p$ annihilates $B_{n-1}(P)$ and $\eta_{n-1}$ is onto, by 2.1 there is a $k: h_{n-1}(P)$ $\rightarrow C_{n-1}(K)$ with $k \eta_{n-1}=\varphi_{n-1}-\partial^{\prime} p$. Thus $\varphi_{+} \eta_{n-1}=\eta^{\prime} k \eta_{n-1}$ hence $\varphi_{+}=\eta^{\prime} k$ and, because $C_{n-1}(K)$ is $W$-free, the desired factoring is obtained.

From 4.4, it follows that a map with degree zero induces the zero homomorphism $H_{n}(P) \rightarrow H_{n}(K)$; its behavior for the homomorphism $h_{n}(P) \rightarrow h_{n}(K)$ is somewhat different. This is given in

5.4. Theorem. Let $P, K$ have operators $W$, and let $\varphi: P^{n+1} \rightarrow K^{n+1}$ be an equivariant chain map. Assume $(\mathrm{a}): C_{n-1}(P)$ is $W$-free and $(\mathrm{b}): e_{W}^{n}(\varphi)=0$. Then the homomorphism $\varphi_{+}: h_{n}(P) \rightarrow h_{n}(K)$ factors into $h_{n}(P) \rightarrow F \rightarrow h_{n}(K)$ where $F$ is $W$-free.

Proof. By hypothesis, $\eta \varphi_{n}=q \partial$ for some $W$-homomorphism $q: C_{n-1}(P)$ $\rightarrow h_{n}(K)$. As in 5.2 , there is a $W$-homomorphism $p: C_{n-1}(P) \rightarrow C_{n}(K)$ with 
$\eta p=q$. Furthermore, using the diagram

$$
C_{n-1}(P) \stackrel{\partial}{\leftarrow} C_{n}(P) \stackrel{\bar{\eta}}{\rightarrow} h_{n}(P)
$$

and 2.1 , there is an $l: h_{n}(P) \rightarrow C_{n-1}(P)$ with $l \bar{\eta}=\partial$. Thus, $\eta \varphi_{n}=\eta p l \bar{\eta}$; this shows $\eta p l$ is the homomorphism $h_{n}(P) \rightarrow h_{n}(K)$ induced by $\varphi$ and the assertion follows.

The following corollaries are immediate:

5.5. Corollary. Let $P, K$ have operators $W$, and let $\varphi, \psi: P^{n+1} \rightarrow K^{n+1}$ be equivariant chain maps. If both $C_{n-1}(P)$ and $C_{n-2}(P)$ are $W$-free, and if $e_{W}^{n-1}(\varphi)$ $=e_{W}^{n-1}(\psi)$ then $e_{W}^{n}(\varphi)-e_{W}^{n}(\psi) \in \Delta_{W}^{n}\left(P ; H_{n}(K)\right)$.

Proof. Applying 5.4 to the chain map $\varphi-\psi$, the equality of their $(n-1, W)$ degrees gives a factoring of $(\varphi-\psi)_{+}: h_{n-1}(P) \rightarrow h_{n-1}(K)$ and therefore 5.2 applies.

5.6. Corollary. Let $C_{n-1}(K)$ be $W$-free. Then $e_{W}^{n}(K) \in \Delta_{W}^{n}\left(K ; H_{n}(K)\right)$ if and only if the identity automorphism of $h_{n-1}(K)$ factors into $h_{n-1}(K) \rightarrow F$ $\rightarrow h_{n-1}(K)$ where $F$ is $W$-free.

Proof. $e_{W}^{n}(K)$ is the $(n, W)$-degree of the identity map of $K$.

5.7. Corollary. Let $W$ operate simply on $K$. Then $e^{n}(K) \in \Delta^{n}\left(K ; H_{n}(K)\right)$ $\approx H^{n}\left(K ; H_{n}(K)\right)$ if and only if $H_{n-1}(K)$ is free.

Proof. Observe first that the factoring $h_{n-1} \rightarrow F \rightarrow h_{n-1}, F$ free, of the identity automorphism of $h_{n-1}$ exists if and only if $h_{n-1}$ is free, since such a factoring implies at once that $h_{n-1}$ is isomorphic to a direct summand of the free group $F$. Furthermore $h_{n-1}=H_{n-1} \oplus M$ and since $M$ is isomorphic to the subgroup $B_{n-2}(K)$ of the (free) group $C_{n-2}(K), M$ is free. Thus, $h_{n-1}$ is free if and only if $H_{n-1}$ is free. The result follows from 5.6.

5.8. Corollary. Let $W$ operate simply. Then $e^{n}(\varphi) \in \Delta^{n}\left(P ; H_{n}(K)\right)$ $\approx H^{n}\left(P ; H_{n}(K)\right)$ if and only if $\varphi_{+}: H_{n-1}(P) \rightarrow H_{n-1}(K)$ factors into $H_{n-1}(P)$ $\rightarrow F \rightarrow H_{n-1}(K)$ where $F$ is free.

Proof. Immediate from the decomposition $h_{n-1}=H_{n-1} \oplus M$ and 5.2, 5.3.

6. Spaces with operators. The discussion thus far has been purely algebraic. Consider now a topological space $Y$ with a group $W$ of continuous transformations $w: Y \rightarrow Y$. The total singular complex [4, p. 187] $S(Y)$ is then an augmentable complex and each $w$ induces an isomorphism $w_{s}$ : $S(Y) \rightarrow S(Y) ; S(Y)$ thus appears as a complex with operators $W$. Setting $h_{n}(Y)=C_{n}(Y) / B_{n}(Y)$, the singular integral $n$-chains modulo the singular $n$ boundaries, $h_{n}(Y)$ is a topological invariant of $Y$ and the results $\left(^{8}\right)$ of $\S \S 2-5$

(8) By [3, p. 65] $C_{n}(Y)$ is $W$-free if and only if no transformation $w: Y \rightarrow Y$, other than $w=1$ has a fixed point. 
are valid with the complexes replaced by topological spaces and continuous equivariant maps taking the place of chain maps throughout.

If $Y$ is a polytope $P$ in a fixed simplicial subdivision, there are two ways in which to arrive at $h_{n}(P)$, and hence at $H_{W}^{l}\left(X ; h_{n}(P)\right)$ : to work in the singular complex $S(P)$ or else to work in the complex $k(P)$ determined by $P$ itself. The two groups $h_{n}(S(P))$ and $h_{n}(k(P))$ are clearly not the same, and consequently for any space $X, H_{W}^{l}\left(X ; h_{n}(S(P))\right)$ and $H_{W}^{l}\left(X ; h_{n}(k(P))\right)$ differ in general. Despite this, it will be shown that the groups $\Delta_{W}^{l}\left(X ; H_{n}(S(P))\right)$ and $\Delta_{W}^{l}\left(X ; H_{n}(k(P))\right)$ are always isomorphic. Thus, $\Delta_{W}^{l}$ depends only on $X$ and $P$ as spaces, and on $W$, but not on the particular simplicial subdivision used, and can therefore be computed directly from $k(P)$.

The result stated is obvious if the operators act simply on $X$ and $P$, because $H_{n}(P)$ is a direct summand of $h_{n}(P)$ and consequently $\Delta^{l}\left(X ; H_{n}(P)\right)$ $\approx H^{l}\left(X ; H_{n}(P)\right)$. To treat the general case, the following theorem of Eilenberg [2, p. 392] is needed: If the (simplicial) operators $W$ on a polytope $P$ (given in a fixed simplicial subdivision) are regular $\left({ }^{9}\right)$, there are equivariant chain transformations $\alpha: k(P) \rightarrow S(P), \bar{\alpha}: S(P) \rightarrow k(P)$ such that $\alpha \bar{\alpha}$ and $\bar{\alpha} \alpha$ are equivariantly chain homotopic $\left({ }^{10}\right)$ to identity automorphisms.

6.1. Theorem. Let $W$ operate regularly on a polytope $P$ given in a fixed simplicial subdivision, and let $\alpha_{+}: h_{n}(k(P)) \rightarrow h_{n}(S(P))$ be the homomorphism induced by $\alpha$. Then for any space $X$ with operators $W$,

$$
\left(\alpha_{+}\right)^{\sharp}: H_{W}^{l}\left(X ; h_{n}(k(P))\right) \rightarrow H_{W}^{l}\left(X ; h_{n}(S(P))\right)
$$

maps $\Delta_{W}^{l}\left(X ; H_{n}(k(P))\right)$ isomorphically onto $\Delta_{W}^{l}\left(X ; H_{n}(S(P))\right)$.

Proof. Let $k(P)=P^{\prime}$, and $S(P)=P^{\prime \prime}$. That $\left(\alpha_{+}\right)^{*}$ maps $\Delta_{W}^{l}\left(X ; H_{n}\left(P^{\prime}\right)\right)$ onto $\Delta_{W}^{l}\left(X ; H_{n}\left(P^{\prime \prime}\right)\right)$ is immediate from commutativity in the diagram( $\left.{ }^{11}\right)$

$$
\begin{gathered}
H_{W}^{l}\left(X ; H_{n}\left(P^{\prime}\right)\right) \stackrel{\gamma^{\star}}{\rightarrow} H_{W}^{l}\left(X ; h_{n}\left(P^{\prime}\right)\right) \\
\quad\left(\tilde{\alpha}_{+}\right)^{\prime} \downarrow \\
H_{W}^{l}\left(X ; H_{n}\left(P^{\prime \prime}\right)\right) \underset{\tilde{\gamma}^{\sharp}}{\rightarrow} H_{W}^{l}\left(X ; h_{n}\left(P^{\prime \prime}\right)\right)
\end{gathered}
$$

because $\left(\tilde{\alpha}_{+}\right)^{\#}$ is an isomorphism onto. To show $\left(\alpha_{+}\right)^{\#}$ is an isomorphism, let $f: C_{l}(X) \rightarrow H_{n}\left(P^{\prime}\right)$ represent $[f] \in \Delta_{W}^{l}\left(X ; H_{n}\left(P^{\prime}\right)\right)$. If $\left(\alpha_{+}\right)^{*}[f]=0$, then $\alpha_{+} f$ $=q \partial$ for some $W$-homomorphism $q: C_{l-1}(X) \rightarrow h_{n}\left(P^{\prime \prime}\right)$. Because the values

(9) $W$ acts regularly on $P$ if it acts simplicially, and if $w \sigma=\sigma$ for any simplex $\sigma$ implies $w x=x$ for each point $x \in \sigma$. Simplicial $W$ is always regular on the first barycentric subdivision [2, p. 392].

(10) Two equivariant chain maps $\lambda, \mu: P \rightarrow K$ are equivariantly chain homotopic if there is a set of $W$-homomorphisms $D: C_{n}(P) \rightarrow C_{n+1}(K)$ one for each dimension $n$, such that $\lambda c-\mu c$ $=\partial D c-D \partial c$ for every $c \in C_{n}(P)$ and every $n$.

(11) $\tilde{\alpha}_{+}$represents the isomorphism $H_{n}\left(P^{\prime}\right) \rightarrow H_{n}\left(P^{\prime \prime}\right)$ induced by $\alpha$, and $\gamma$ the injection $H_{n} \rightarrow h_{n}$. 
of $f$ are always in $H_{n}\left(P^{\prime}\right)$ it follows that $\bar{\alpha}_{+} \alpha_{+} f=f$ hence $f=\bar{\alpha}_{+} q \partial$; since $\bar{\alpha}_{+} q: C_{l-1}(X) \rightarrow h_{n}\left(P^{\prime}\right)$ is $W$-equivariant, this shows $[f]=0$ in $\Delta_{W}^{l}\left(X ; H_{n}\left(P^{\prime}\right)\right)$ proving the result.

Regarding the $(n, W)$-universal cohomology class of a polytope,

6.2. TheOREM. Let $W$ operate regularly on the polytope $P$. In the string of homomorphisms

$$
H_{W}^{n}\left(k(P) ; h_{n}(k(P))\right) \stackrel{\bar{\alpha}_{+}}{\longrightarrow} H_{W}^{n}\left(S(P) ; h_{n}(k(P))\right) \stackrel{\left(\alpha_{+}\right)^{*}}{\longrightarrow} H_{W}^{n}\left(S(P) ; h_{n}(S(P))\right)
$$

the image of $e_{W}^{n}(k(P))$ is $e_{W}^{n}(S(P))$.

Proof. Using 4.2, it follows easily that the image of $e_{W}^{n}(k(P))$ in this string of homomorphisms is represented by $\eta \alpha \bar{\alpha}$, where $\eta: C_{n}(S(P)) \rightarrow h_{n}(S(P))$ is the natural projection. Let $D: C_{n}(S(P)) \rightarrow C_{n+1}(S(P))$ be the equivariant chain homotopy of $\alpha \bar{\alpha}$ to the identity, so that $\alpha \bar{\alpha}-1=\partial D-D \partial$. Then $\eta \alpha \bar{\alpha}-\eta=-\eta D \partial$ showing $\eta \alpha \bar{\alpha}$ cohomologous to $\eta$, completing the proof.

Thus, under the homomorphism indicated in 6.2, the universal $(n, W)$ classes of $k(P)$ and $S(P)$ always correspond to one another.

II.

7. A universal coefficient theorem for mappings. Let $P, K$ be two complexes, and let $W$ operate simply. It is well-known that $[1$, p. 238] whenever $H_{n-1}(P)$ is free, two maps inducing identical homomorphisms $H_{n}(P) \rightarrow H_{n}(K)$ induce identical homomorphisms of all homology and cohomology groups. This result is a consequence of the following more general theorem which applies also to the case that $W$ does not necessarily operate simply:

7.1. Theorem. Let $P$ and $K$ be complexes with operators $W$. Assume (a): $C_{n-1}(P)$ is $W$-free, and (b): The identity automorphism of $h_{n-1}(P)$ factors into $h_{n-1}(P) \rightarrow F \rightarrow h_{n-1}(P)$ where $F$ is $W$-free. Then two maps $\varphi, \psi: P \rightarrow K$ inducing the same homomorphism $H_{n}(P) \rightarrow H_{n}(K)$ induce identical homomorphisms for all homology, cohomology, and W-equivariant cohomology groups.

Proof. According to 5.6, $e_{W}^{n}(P)$ is representable by an $\left(n, H_{n}(P)\right)$ cocycle $f$. Using 4.2, $e_{W}^{n}(\varphi)=\left(\varphi_{+}\right)^{*}[f]$ and $e_{W}^{n}(\psi)=\left(\psi_{+}\right)^{t}[f]$; since $\varphi_{+}, \psi_{+}: H_{n}(P) \rightarrow H_{n}(K)$ are the same, it follows that $e_{W}^{n}(\varphi)=e_{W}^{n}(\psi)$. Noting further that hypothesis (b) implies $H_{n-1}(P)$ is free, $4.3,4.6$ give the result.

8. A universal coefficient theorem for equivariant cohomology groups. It is known that $W, G, H_{n}$ and $H_{n-1}$ do not suffice to determine $H_{W}^{n}(K ; G)$. In this section, it will be shown that $H_{W}^{n}(K ; G)$ is an extension of a group determined by $G, W$, and $h_{n-1}$, by a group determined by $G, W, h_{n}$ and $H_{n}$, whenever $C_{n-1}$ is $W$-free.

Let $K$ be a complex with operators $W$, and for any $W$-group $G$ define the homomorphism $\left({ }^{12}\right)$

(12) For $W$-groups $A, B$, $\operatorname{Hom}_{W}(A, B)$ is the group of all $W$-homomorphisms $A \rightarrow B$. 


$$
\theta: \operatorname{Hom}_{W}\left(h_{n}(K), G\right) \rightarrow H_{W}^{n}(K ; G)
$$

by $\theta(p)=p^{\#} e_{W}^{n}(K)=[p \eta]$.

8.1. $\theta$ is onto, and $p \in$ kernel $\theta$ if and only if $p \eta=\mu \partial$ for some

$$
\mu \in \operatorname{Hom}_{W}\left(C_{n-1}(K), G\right) .
$$

That $\theta$ is onto follows from 3.2 , and the rest is trivial.

To investigate kernel $\theta$, the homomorphism $\partial$ of $C_{n}(K)$ onto $B_{n-1}(K)$ is "lifted" to one of $h_{n}(K)$ onto $B_{n-1}(K)$ :

8.2. There is a homomorphism $\zeta$ of $h_{n}(K)$ onto $B_{n-1}(K)$ such that $\zeta \eta=\partial$ and $p \in$ kernel $\theta$ if and only if $p=\mu \zeta$ for some $\mu \in \operatorname{Hom}_{W}\left(C_{n-1}(K), G\right)$.

Consider the diagram

$$
B_{n-1}(K) \stackrel{\partial}{\leftarrow} C_{n}(K) \stackrel{\eta}{\rightarrow} h_{n}(K) .
$$

Here, $\eta$ is onto, and kernel $\eta=B_{n} \subset Z_{n}=$ kernel $\partial$, hence 2.1 gives the required homomorphism. It is clear that $\zeta$ is onto, and the rest follows from 8.1.

The homomorphism $\zeta^{+}: \operatorname{Hom}_{W}\left(B_{n-1}(K), G\right) \rightarrow \operatorname{Hom}_{W}\left(h_{n}(K), G\right)$ induced by $\zeta$ is now studied. Let $\gamma^{+}: \operatorname{Hom}_{W}\left(h_{n}(K), G\right) \rightarrow \operatorname{Hom}_{W}\left(H_{n}(K), G\right)$ be the homomorphism induced by the injection $\gamma: H_{n}(K) \rightarrow h_{n}(K)$. Then

8.3. $\zeta^{+}$is an isomorphism onto the subgroup kernel $\gamma^{+}$.

That $\zeta^{+}$is an isomorphism into is immediate because $\zeta$ is onto. To find the image, consider first $\zeta^{+}(f)$. For any $z \in Z_{n}(K)$ one has $\zeta^{+}(f) \eta z=f \zeta \eta z=f \partial z=0$ hence $\zeta^{+}(f):\left(h_{n}, H_{n}\right) \rightarrow(G, 0)$. Conversely, if $f:\left(h_{n}, H_{n}\right) \rightarrow(G, 0)$ then in the diagram

$$
B_{n-1} \stackrel{\zeta}{\leftarrow} h_{n} \stackrel{f}{\rightarrow} G
$$

$\zeta$ is onto, and kernel $\zeta=H_{n} \subset$ kernel $f$, so 2.1 gives a $W$-homomorphism $f^{\prime}: B_{n-1} \rightarrow G$ with $f=f^{\prime} \zeta=\zeta^{+}\left(f^{\prime}\right)$. The image group is therefore exactly kernel $\gamma^{+}$.

Let $i^{+}: \operatorname{Hom}_{W}\left(C_{n-1}(K), G\right) \rightarrow \operatorname{Hom}_{W}\left(B_{n-1}(K), G\right)$ be the homomorphism induced by the injection $i: B_{n-1}(K) \rightarrow C_{n-1}(K)$. Then

8.4. Kernel $\theta=\zeta^{+} i^{+} \operatorname{Hom}_{W}\left(C_{n-1}(K), G\right)$.

This is immediate from 8.2.

It follows from $8.2,8.4$ that

$$
H_{W}^{n}(K ; G)=\operatorname{Hom}_{W}\left(h_{n}, G\right) / \zeta^{+} i^{+} \operatorname{Hom}_{W}\left(C_{n-1}, G\right) .
$$

Now,

$$
\frac{\operatorname{Hom}_{W}\left(h_{n}, G\right)}{\zeta^{+} i^{+} \operatorname{Hom}_{W}\left(C_{n-1}, G\right)} / \frac{\zeta^{+} \operatorname{Hom}_{W}\left(B_{n-1}, G\right)}{\zeta^{+} i^{+} \operatorname{Hom}_{W}\left(C_{n-1}, G\right)} \approx \frac{\operatorname{Hom}_{W}\left(h_{n}, G\right)}{\operatorname{Kernel} \gamma^{+}} \approx \text { Image } \gamma^{+} \text {. }
$$

If $C_{n-1}$ is $W$-free, then $\operatorname{Hom}_{W}\left(B_{n-1}, G\right) / i^{+} \operatorname{Hom}_{W}\left(C_{n-1}, G\right)$ is readily verified, as in $\left[4\right.$, p. 161], to be essentially a function of $W, G$, and $C_{n-1} / B_{n-1}$; it is 
written $\operatorname{Ext}_{W}\left(h_{n-1}(K), G\right)$. This leads at once to the universal coefficient theorem:

8.5. THEOREM. If $C_{n-1}(K)$ is $W$-free, then there are homomorphisms $\alpha, \beta$, such that the string

$$
0 \rightarrow \operatorname{Ext}_{W}\left(h_{n-1}(K), G\right) \stackrel{\alpha}{\rightarrow} H_{W}^{n}(K ; G) \stackrel{\beta}{\rightarrow} \text { Image } \gamma^{+} \rightarrow 0
$$

is exact.

That this reduces to the usual universal coefficient theorem when $W$ operates simply, is easily seen by using the decomposition $h_{n}=H_{n} \oplus B_{n-1}$ in the initial expression for $H^{n}(K ; G)$.

It is trivial to verify that $\alpha, \beta$ are "natural" under equivariant chain maps of one complex into another. This leads to another universal coefficient theorem for mappings, analogous to that in $[4$, p. 161].

9. Application to the obstruction cocycle. Let $P, K$ be complexes with operators $W$, and $Q \subset P$ a segregated subcomplex. For each equivariant $\phi: Q \cup P^{n} \rightarrow K$ Eilenberg and MacLane [3, p. 57] have defined a (homology) $(n+1)$-obstruction cocycle $l^{n+1}(\phi)$ by setting

$$
l^{n+1}(\phi)=\eta \phi \partial: C_{n+1}(P) \rightarrow H_{n}(K) .
$$

Clearly $l^{n+1}(\phi)=0$ on $Q$, hence it represents an element

$$
a_{W}^{n+1}(\phi) \in H_{W}^{n+1}\left(P, Q ; H_{n}(K)\right) .
$$

The fundamental property of $a_{W}^{n+1}(\phi)$ is [3, p. 58].

9.1. If the pair $\left(C_{i}(P), C_{i}(Q)\right)$ is $W$-free $\left({ }^{13}\right)$ for $i=n, n+1$, then $a_{W}^{n+1}(\phi)=0$ if and only if there is an equivariant extension of $\phi \mid Q \cup P^{n-1}$ over $Q \cup P^{n+1}$.

The question arises: Suppose $a_{W}^{n+1}(\phi) \neq 0$; is it true that $\phi \mid Q \cup P^{n-i}$ for some $i \geqq 2$, is nevertheless extendable over $Q \cup P^{n+1}$ ? Put another way: given a map $\varphi: Q \rightarrow K$ known to be extendable over $Q \cup P^{n}$; is it possible to select an extension $\phi$ so that $a_{W}^{n+1}(\phi)=0$ ? The application here is to give the mechanism whereby the map $\varphi$ on $Q$ itself predetermines the set of obstructions $\left\{a_{W}^{n+1}(\phi)\right\}$ that can be met by its extensions over $Q \cup P^{n}$.

9.2. Definition. The $(n+1)$-extension degree of the equivariant chain $\operatorname{map} \varphi: Q \rightarrow K$ is the element

$$
\delta^{+} e_{W}^{n}(\varphi) \in H_{W}^{n+1}\left(P, Q ; h_{n}(Y)\right),
$$

where $\delta^{+}: H_{W}^{n}(Q, \cdot) \rightarrow H_{W}^{n+1}(P, Q ; \cdot)$ is the coboundary homomorphism.

Observe that the $(n+1)$-extension degree is defined without reference to any extension of the chain map $\varphi$.

Now let $\gamma^{\sharp}: H_{W}^{n+1}\left(P, Q ; H_{n}(Y)\right) \rightarrow H_{W}^{n+1}\left(P, Q ; h_{n}(Y)\right)$ be the homomorphism

(13) If $G$ is $W$-free and $H$ is a subgroup of $G$, the couple $(G, H)$ is termed $W$-free if there is a $W$-basis $\left\{g_{\mu}\right\}$ for $G$ such that $\left\{g_{\mu}\right\} \cap H$ is a $W$-basis for $H$. 
induced by the injection $\gamma: H_{n}(Y) \rightarrow h_{n}(Y)$. The following theorem provides a connection between the $(n+1)$-extension degree of $\varphi$ and the $(n+1)$ obstruction of an extension $\phi: Q \cup P^{n} \rightarrow K$ of $\varphi$ :

9.3. Theorem. Let $\varphi: Q \rightarrow K$ be an equivariant chain map, and $\phi: Q \cup P^{n} \rightarrow K$ any equivariant extension. Then

$$
\gamma^{*} a_{W}^{n+1}(\phi)=\delta^{+} e_{W}^{n}(\phi) .
$$

Proof. Recall that $\delta^{+} e_{W}^{n}(\varphi)$ is represented by the $\bmod Q$ cocycle $f \partial$ where $f$ is any equivariant extension of $\eta \varphi \in Z_{W}^{n}\left(Q ; h_{n}(K)\right)$ to an $f \in C_{W}^{n}\left(P ; h_{n}(K)\right)$. It is clear that $f-\eta \phi$ is zero on $Q$, hence $f-\eta \phi$ is an $n$-cochain in $P-Q$. Since

$$
(f-\eta \phi) \partial=f \partial-l^{n+1}(\phi)
$$

this shows $f \partial$ cohomologous to $l^{n+1}(\phi)$ in $Z_{W}^{n+1}\left(P, Q ; h_{n}(K)\right)$ and proves the theorem.

Since $\delta^{+} e_{W}^{n}(\varphi)$ is defined completely independently of any extension of $\varphi$, 9.3 says that if an extension of $\varphi$ over $Q \cup P^{n}$ is possible, then $\delta^{+} e_{W}^{n}(\varphi)$ must lie in $\Delta_{W}^{n+1}\left(P, Q ; H_{n}(K)\right)$ and, indeed, that the $(n+1)$-obstruction cocycles that will be met by any extension of $\varphi$ will represent some element of $\gamma^{\sharp-1} \delta^{+} e_{W}^{n}(\varphi)$. The set of all $(n+1)$-obstructions that can possibly be met by an extension of $\varphi$ over $Q \cup P^{n}$ are therefore determinable in advance.

The theorem has a particularly simple consequence for continuous maps of a polytope $P$ into an $n$-simple space $Y$ when the operators act simply. With each continuous $F: Q \cup P^{n} \rightarrow Y$ Eilenberg [5, p. 149] associates a (homotopy) (n+1)-obstruction cocycle $c^{n+1}(F) \in Z^{n+1}\left(P, Q ; \pi_{n}(Y)\right)$. It is trivial to verify that under the Hurewicz homomorphism $\chi: \pi_{n}(Y) \rightarrow H_{n}(Y)$, $\chi^{\sharp}\left[c^{n+1}(F)\right]=a^{n+1}(F)$.

9.4. Corollary. Let $f: Q \rightarrow Y$ be extendable to a continuous $F: Q \cup P^{n} \rightarrow Y$. If $\chi^{\sharp}: H^{n+1}\left(P, Q ; \pi_{n}(Y)\right) \rightarrow H^{n+1}\left(P, Q ; H_{n}(Y)\right)$ is an isomorphism (into or onto) then any other extension $F^{\prime}$ of $f$ over $Q \cup P^{n}$ will have $c^{n+1}\left(F^{\prime}\right)$ cohomologous to $c^{n+1}(F)$. That is, no alteration of the class of the $(n+1)$-obstruction initially met is possible without altering $f$ itself.

Proof. According to $9.3, \gamma^{t} \chi^{t}\left[c^{n+1}(F)\right]=\delta^{+} f^{+} e_{W}^{n}(Y)$. Since the operators act simply, $\gamma^{\sharp}$ is an isomorphism into, hence so also is $\gamma^{\sharp} \chi^{\sharp}$.

\section{BiBLIOGRAPHY}

1. P. Alexandroff and H. Hopf, Topologie, Berlin, Springer, 1935.

2. S. Eilenberg, Homology of spaces with operators, Trans. Amer. Math. Soc. vol. 61 (1947) pp. $378-417$.

3. S. Eilenberg and S. MacLane, Homology of spaces with operators, II, Trans. Amer. Math. Soc. vol. 65 (1949) pp. 49-99.

4. S. Eilenberg and N. Steenrod, Foundations of algebraic topology, Princeton University Press, 1952.

5. N. Steenrod, The topology of fibre bundles, Princeton University Press, 1951.

The University of Southern California 